\title{
ARUTE EPIGIOTIIIS IN ADULTS
}

\section{DR. S. ALMERIE}

Emergency Medicine Specialist

Linclonshire Hospital Trust

United Kingdom

Key words: Epiglottitis, Haemophilus.

Most clinicians would be aware of this disease, which is rare in children but even rarer in adult. The evolving epidemiology in the last 2 decades (HIB vaccination programme), means that clinicians need to increase their vigilance in diagnosing this condition in adults.

Epiglottitis in adults is a rare disease and fatal if not treated in a timely manner. Incidence is about 1 case per 100,000 people per year. This report highlights the need for quick identification of this situation and subsequent successful treatment, which results in saving the patient's life. It is more frequently a male disease, occurring during the fifth decade of life, and its occurrence has decreased dramatically since the introduction of Haemophilus influenza type $b$ vaccine. The onset and progression of symptoms of epiglottitis is rapid (George Washington woke up with a sore throat and died that night).

Epiglottitis in adults is, as stated, a rare disease but fatal if not treated quickly-being an acute inflammation in the supraglottic region of the oral pharynx with inflammation of the epiglottis, vallecula, arytenoids, and aryepiglottic folds. Risk of death for persons with epiglottitis is high due to sudden airway obstruction and consequential difficulty in intubating patients with extensive swelling of supraglottic structures. The average age among adults contracting the disease is 45 years.

\section{CASE REPORTING}

A 51 year old female with past medical history of paroxysmal SVT, depression on B-blocker, sotalol, citalopram and lansoprazole, attended the out of hours GP services in the early hours of the morning complaining of a sore throat which started 2-3 hours ago. At that time she was stable and apyrexial with a temperature $(T)$ of $36.3^{\circ} \mathrm{C}$, pulse rate (PR) was $77 / \mathrm{min}$,
$\mathrm{SaO} 2=99 \%$ on air and respiratory rate (RR) of $14 / \mathrm{min}$. On examination nothing abnormal was found and she was discharged with diagnosis of laryngitis and advice.

Three hours later, the patient attended the Accident and Emergency department (A\&E) complaining of swelling to her throat and difficulty in swallowing. On initial assessment the patient looked unwell and short of breath, unable to complete full sentences. Her initial observations were:

$$
\begin{array}{ll}
\text { - } & \mathrm{P}=125 / \mathrm{min} . \\
\text { - } & \mathrm{B} / \mathrm{P}=167 / 96 . \\
\text { - } & \mathrm{RR}=16 / \mathrm{min} . \\
\text { - } & \mathrm{T}=37^{\circ} \mathrm{C} . \\
\text { - } & \mathrm{SaO} 2=99 \% . \\
\mathrm{GCS}=15 / 15 .
\end{array}
$$

On examination there was audible inspiratory stridor, trachea was central, there was an inspiratory wheeze to the chest, no lymphoadenopathy, no drooling.

She denied any history of any foreign body (FB) or any trauma.

The impression was epiglottits.

She was taken immediately into the resuscitation room.

The anaesthetic and the Ear Nose and Throat (E.N.T) consultants were fast bleeped.

For treatment she had high flow 02,salbutamol 500 microgram via nebuliser, adrenaline via nebuliser, was cannulated,blood was sent, including blood cultures. She received IV hydrocotisone, adrenalin e1:1000 IM , and IV cefotaxime $2 \mathrm{~g}$. In 10 minutes, her observations were repeated Pulse $=102 / \mathrm{min}, B / P=130 / 70$, 
$\mathrm{RR}=14 / \mathrm{min}, \mathrm{T}=37^{\circ} \mathrm{C}, \mathrm{SO} 2=99 \%, \mathrm{GCS}=15 / 15$, and the patient was feeling better.

The patent was transferred to the Intensive Care Unit/ High Dependency Unit (ICU/HDU) where a laryngoscopy was performed and the diagnosis confirmed as inflamed epiglottis and supra glottis.

Her blood results came as $W C C=20, \quad C R P=38$, NEUTROPHILS=17.30 with other blood tests showing as normal.

Swab results were negative.

Blood cultures were negative.

After continuing the treatment plan, including close observation to the airway, the patient was gradually feeling much better and the strider disappeared. Within the next 24 hours, the patient was able to eat soft diet and after 4 days she was discharged home with cefalexine $500 \mathrm{mg}$ TDS, and out patient follow up.

\section{DISCUSSION}

Epiglottitis is an acute inflammation in the supraglottic region of the oropharynx with inflammation of the epiglottis, vallecula, arytenoids, and aryepiglottic folds. In adults with acute epiglottitis, blood cultures for $\mathrm{H}$ influenza are positive in about $25 \%$ of cases. In remaining adult cases, Haemophilus parainfluenzae, Streptococcus pneumonia, and group A streptococci are frequently isolated from pharyngeal cultures. Less common infectious aetiologies include other bacteria (eg, Staphylococcus aureus, mycobacterium).

Physical findings of epiglottitis may include the following: unwell, acute fever, drooling or inability to handle secretions cervical adenopathy and stridor.

Late findings indicating advanced airway obstruction include muffled voice, tripod position, respiratory distress mild cough ,fever , irritability ,tachycardia, and a general toxic appearance of the patient.

As a differential diagnosis we should think about abscess, toxicity, airway obstruction, foreign body aspiration, bacterial laryngotracheobronchitis, and acute angioedema.

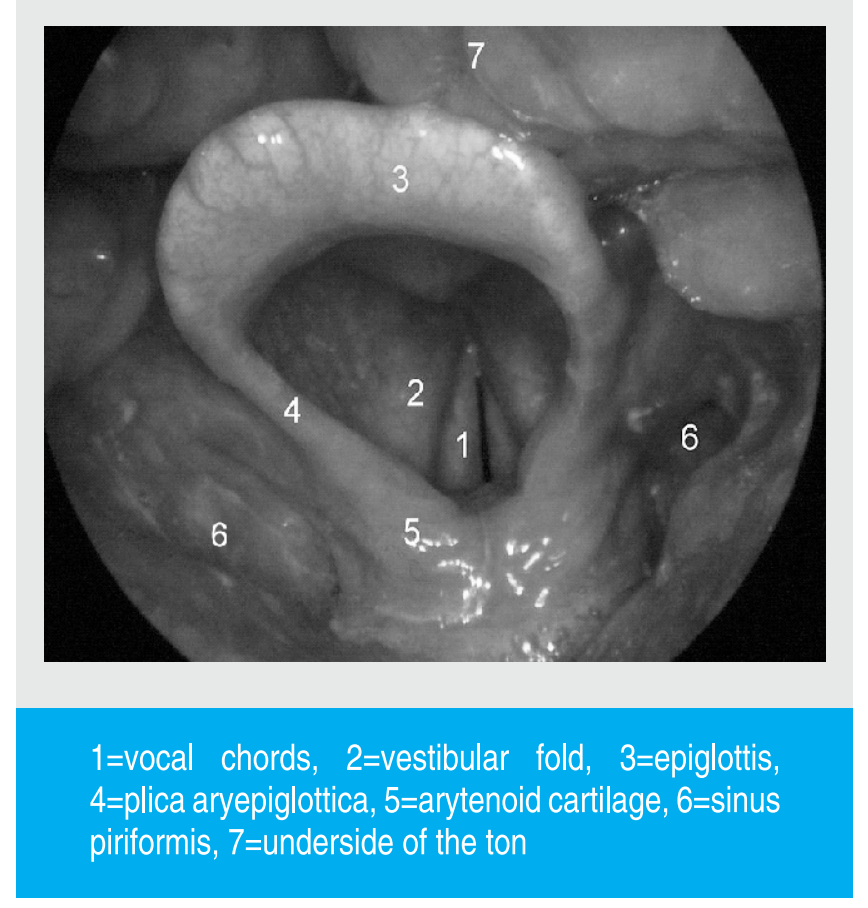

\section{INVESTIGATIONS}

- $\quad$ Epiglottic swabs may be taken when the airway is secured or capable of being secured rapidly if needed.

- $\quad$ Blood cultures may be taken, particularly if the patient is systemically unwell.

- Blood samples.

\section{Emergency treatment}

Signs and symptoms associated with a need for intubation include respiratory distress, airway compromise on exam, stridor, inability to swallow, drooling, sitting erect, and deterioration within 8-12 hours. Enlarged epiglottis on radiographs is associated with airway obstruction. When in doubt, securing the airway is likely to be the safest approach. Patients without any signs of airway compromise, respiratory difficulty, stridor, or drooling, and who have only mild swelling on laryngoscope may be managed without immediate airway intervention by close monitoring in the ICU.

- $\quad$ Avoid agitating the patient with acute epiglottitis. Administer supplemental humidified oxygen if possible, but do not force the patient, as the 
resultant agitation could worsen the condition.

- $\quad$ Equipment for intubation should be available at the bedside.

- $\quad$ An anaesthesiologist and an ENT specialist or a general surgeon should be notified as soon as possible.

Epinephrine, corticosteroids, and beta-agonists have not been proven to be helpful. Corticosteroid usage remains controversial, although anecdotal reports in the past had supported its use. Adrenaline nebulisers may help. Empiric coverage for group Streptococcus pneumonia,
Staphylococcus pyogenes, and $\mathrm{H}$ influenza should be provided (a third-generation cephalosporin or amoxicillin/clavulanic acid). Third-generation cephalosporin are preferred as first-line agents because of increasing resistance to ampicillin.

\section{Copyright@ 03 Sep, 2011.}

\section{REFERENCES}

1. Jeffrey Glenn Bowman, MD, MS, Consulting Staff, Highfield MRI, Columbus, Ohio.

2. Oxford emergency medicine,2008.
Correspondence Address:

Dr. S. Almerie

Emergency Medicine Specialist

Linclonshire Hospital Trust

United Kingdom

salmerie@hotmail.co.uk

\section{Article Citation:}

Almerie S. Acute epiglottits in adult. Professional Med J Dec 2011;18(4): 722-724.

\section{PREVIOUS RELATED STUDIES}

\section{A CREATIVE MAN IS MOTIVATED BY THE DESIRE TO ACHIEVE, NOT BY THE DESIRE TO BEAT OTHERS.} $\underline{\text { AYN RAND }}$ 